\title{
ANÁLISIS COMPARATIVO DE ÍNDICES DE SEQUÍA EN ANDALUCÍA PARA EL PERIODO 1901-2012
}

\author{
M. PEÑA-GALLARDO ${ }^{1}$, S.R. GÁMIZ-FORTIS ${ }^{2}$, \\ Y. CASTRO-DÍEZ ${ }^{2}$, M.J. ESTEBAN-PARRA ${ }^{2 *}$ \\ ${ }^{1}$ Instituto Pirenaico de Ecología, Consejo Superior de Investigaciones Científicas (IPE-CSIC), \\ Zaragoza, España. \\ ${ }^{2}$ Departamento de Física Aplicada, Facultad de Ciencias, Universidad de Granada, España.
}

\begin{abstract}
RESUMEN. El estudio del comportamiento temporal de la sequía a partir de series meteorológicas constituye una importante herramienta de actuación y prevención ante este fenómeno natural de carácter extremo, que ocasiona importantes impactos económicos, sociales y medioambientales. Como buena parte de los fenómenos climáticos, su carácter inhabitual y anómalo hace que definir y ubicar espacialmente el riesgo de sequía resulte una difícil tarea, más aún en las llamadas regiones de transición, como las dominadas por el clima mediterráneo, caracterizadas por una elevada variabilidad pluviométrica. Junto a ello, la realidad que supone la existencia de un cambio climático lleva al interés por conocer el comportamiento de las precipitaciones, puesto que de él dependerá el desarrollo y posible intensificación de las condiciones secas futuras.
\end{abstract}

El objetivo global de este trabajo es el análisis de la detección y evolución de los episodios de las sequías en Andalucía en el período 1901-2012, caracterizadas mediante tres índices de sequía diferentes: el Índice Estandarizado de Precipitación (SPI), el Índice Estandarizado de Precipitación y Evapotranspiración (SPEI) y el Índice Estandarizado de Sequía Pluviométrica (IESP), calculados en tres ventanas temporales del periodo inicial 1901-2012. Este análisis se ha llevado a cabo después de un estudio preliminar de las tendencias de precipitación con la intención de entender el comportamiento de las mismas, ya que esta variable climática es una de las más importantes en el análisis de extremos. Los objetivos particulares de este estudio son: (1) investigar y caracterizar las sequías meteorológicas en Andalucía, principalmente los episodios más importantes; (2) proporcionar una evaluación global de las capacidades de cada uno de los tres índices analizados para caracterizar la sequía en un territorio climáticamente heterogéneo; y (3) describir el comportamiento de las series precipitación y de los índices de sequía considerados, con el fin de establecer las características generales de la evolución de los mismos en Andalucía. Los resultados han 
demostrado que no todos los índices responden de la misma manera en la identificación de la intensidad y duración de los periodos de sequía en este tipo de región en la que la variabilidad geográfica y climática es uno de los elementos principales a considerar.

\section{Comparative analysis of drought indices in Andalusia during the period 1901-2012}

ABSTRACT. The aim of this paper is the analysis of the detection and evolution of droughts occurred in Andalusia for the period 1901-2012, by applying three different drought indices: the Standardized Precipitation Index (SPI), the Standardized Precipitation and Evapotranspiration Index (SPEI) and the Standardized Drought-Precipitation Index (IESP), computed for three time windows from the initial period 1901-2012. This analysis has been carried out after a preliminary study of precipitation trends with the intention of understanding the precipitation behaviour, because this climatic variable is one of the most important in the study of extreme events. The specific objectives of this study are: (1) to investigate and characterize the meteorological drought events, mainly the most important episodes in Andalusia; (2) to provide a global evaluation of the capacities of the three different considered indices in order to characterize the drought in a heterogeneous climatically territory; and (3) to describe the temporal behaviour of precipitation and drought indices series in order to establish the general characteristics of their evolution in Andalusia. The results have shown that not all the indices respond similarly identifying the intensity and duration of dry periods in this kind of region where geographical and climatic variability is one of the main elements to be considered.

Palabras clave: índices de sequía, precipitación, Andalucía, SPI, SPEI, IESP.

Key words: drought indices, precipitation, Andalusia, SPI, SPEI, IESP.

Recibido el 30 de enero de 2016

Aceptado el 14 de marzo de 2016

*Correspondencia: Departamento de Física Aplicada, Facultad de Ciencias. Universidad de Granada. Campus de Fuentenueva. 18071 Granada, España. E-mail: esteban@ugr.es

\section{Introducción}

Las sequías han marcado la historia de la humanidad desde tiempos inmemoriales. A pesar de que carecen del aspecto dramático que caracteriza a los terremotos o los huracanes, las sequías causan la muerte y el desplazamiento de más personas que cualquier otro tipo de desastre natural (Jarraud, 2013). Junto a los impactos humanos, este fenómeno climático supone un desastre a nivel ecológico en el sistema natural, entendiendo que a desastre natural conduce 'todo fenómeno extremo del medio físico 
que resulte perjudicial para el hombre y que esté originado por fuerzas externas a él, siendo estas fuerzas incontrolables e imprevisibles, de manera que otorgan al desastre un carácter azaroso que lo convierte en riesgo para la sociedad' (Burton et al., 1979; Pita, 1989).

En el contexto del riesgo, las sequías se catalogan como un riesgo de carácter hidrometeorológico por su origen dicotómico, tanto hidrológico como meteorológico. Están asociadas al decrecimiento o ausencia de precipitación, y pueden definirse en términos meteorológicos, hidrológicos, agrícolas y socioeconómicos, pero siempre como un evento extremo. En la actualidad no se ha alcanzado un acuerdo común en su definición dentro de la comunidad científica (American Meteorological Society, 2004; Wilhite and Glantz, 1985). A pesar de ello, la Organización Meteorológica Mundial en su Vocabulario de Términos Meteorológicos (METEOTERM), la define como una 'ausencia prolongada, deficiencia marcada o pobre distribución de precipitación' o bien como un 'período anormal de tiempo seco, suficientemente prolongado, en el que la falta de precipitación causa un grave desequilibrio hidrológico'. Marzol (2001) señala respecto a estos enfoques que la primera opción hace referencia únicamente a la falta o disminución del volumen de agua precipitada respecto a lo considerado normal en un lugar, mientras que la segunda definición restringe la denominación de sequía a los periodos húmedos en los que no es normal la falta de agua, no contemplando así los meses que son normalmente secos (meses estivales en el contexto de clima templadomediterráneo).

La sequía es pues, uno de los fenómenos climáticos más complejos que afectan a la sociedad y el medio ambiente. Su carácter anómalo y coyuntural, a diferencia de la aridez, así como su ocurrencia en todos los regímenes climáticos, han encauzado la labor científica en esta materia a desarrollar mecanismos y herramientas capaces de proporcionar una evaluación objetiva y cuantitativa de la gravedad de la sequía en un esfuerzo por identificar en el tiempo y en el espacio su inicio, cese, duración, magnitud y extensión superficial. Este esfuerzo investigador ha resultado en la formulación de los índices de sequía, indicadores indirectos basados en datos climáticos entre los que cabe destacar, por su amplio uso, los desarrollados por Palmer en la década de los años sesenta (PDSI, PDI, PHDI, ZINX) (Kangas and Brown, 2007), el formulado por McKee et al. (1995), o el SPI (McKee et al., 1993, 1995; Edwards and McKee, 1997).

Por otro lado, los esfuerzos más interesantes en materia de indicadores de sequía para evaluar su incidencia proceden del desarrollo de nuevos índices tales como el SPEI (Vicente-Serrano et al., 2010; Beguería et al., 2014) o el IESP (Pita, 2001b), este último de específica aplicación en el sistema de monitorización de sequía por parte de la Consejería de Medio Ambiente y Ordenación del Territorio de la Junta de Andalucía. Estos índices pueden diferir a la hora de caracterizar la intensidad y duración de los periodos húmedos o secos y cada uno de ellos puede ser más apropiado en un área determinada. Cabe destacar también que los índices SPI y SPEI presentan la ventaja de poder ser calculados a distintas escalas temporales y permiten la monitorización de diferentes tipos de sequía (Vicente-Serrano et al., 2010; Beguería et al., 2014). 
Estos indicadores de sequía son herramientas de gran importancia en la prevención de impactos sociales y económicos, siéndolo aún más en las regiones donde la variabilidad en el régimen de precipitaciones es la causa primera de su desencadenamiento. Tal es el caso de la Península Ibérica, región geográfica con un predominio de las áreas de transición climática donde las condiciones de sequía poseen un patrón diferenciado debido a su localización intermedia entre un clima templado y subtropical. La precipitación media anual presenta una distribución geográfica muy variada, registrando valores máximos por encima de los $2200 \mathrm{~mm}$ en el noroeste de Navarra y áreas del sudoeste de Galicia, frente a valores mínimos inferiores a los $200 \mathrm{~mm}$ en zonas próximas al Cabo de Gata, en la provincia de Almería (Agencia Estatal de Meteorología e Instituto de Meteorología de Portugal). Además, los climas mediterráneos, en tanto que son climas de transición, se caracterizan precisamente por su elevada variabilidad pluviométrica, hecho que se traduce en la existencia de una irregularidad de la precipitación interanual que, a excepción de la franja norte peninsular, resulta elevada en prácticamente todo el interior del país (Vicente-Serrano, 2006; Cubillo et al., 2007; Marín et al., 2012).

Así, la sequía ha sido en España una problemática que ha preocupado a los investigadores mucho antes de que el cambio climático fuera un tema de actualidad, siendo muchos y variados los trabajos sobre el tema, tales como el avanzado estudio de la repercusión de la sequía en el sureste peninsular realizado a mediados del siglo XIX por Rico (1851). Existen, en el ámbito nacional, numerosos y diversos referentes en investigación que se han enfocado en el estudio de las variaciones y tendencias pluviométricas en la Península Ibérica, así como de la sequía, atendiendo a su incidencia y cuantificación en el territorio a partir de diferentes técnicas como la aplicación de índices. Estos trabajos abarcan periodos temporales muy diversos así como distintas resoluciones espaciales, comprendiendo desde escalas globales a escalas más regionales, como el caso de estudios a nivel de cuenca hidrográfica (Esteban-Parra et al., 1998, 2003; Rodrigo et al., 1999, 2000; Pita, 2001a; Gil et al., 2011; del Río et al., 2011; Ruiz-Sinoga et al., 2012; Ceballos, 2013). En el caso de Andalucía existen trabajos al respecto como el desarrollado por Pita et al. (1999) o Pozo-Vázquez (2000). Este último analizó la influencia de la Oscilación del Atlántico Norte (NAO) sobre la variabilidad de las precipitaciones, obteniendo mediante análisis de regresión, una clasificación de zonas donde la precipitación está más o menos influenciada por esta teleconexión.

Dadas las importantes repercusiones de la sequía, más aún en una zona de alta sobrexplotación hídrica, como es el sur de la Península Ibérica, el análisis de índices como el IESP, el SPI o el SPEI, que permite una identificación precisa de los eventos de sequía, puede ayudar a la monitorización y predicción de la misma.

Así pues, este trabajo se centra en la identificación de sequías en Andalucía, comparando el comportamiento de varios índices y estudiando la evolución temporal de los mismos en el periodo 1901-2012. La escala temporal de análisis es la de 12 meses, asociada a las repercusiones hídricas de la sequía. Se aborda también el estudio de la variabilidad de la precipitación en la región. El artículo se estructura en las siguientes 
partes: el apartado 2 presenta los datos usados así como las principales metodologías empleadas. El apartado 3 muestra los principales resultados del análisis. Finalmente, el apartado 4 resume las principales conclusiones del mismo.

\section{Materiales y métodos}

\section{1. Área de estudio}

Andalucía se sitúa en una latitud comprendida entre $36^{\circ} \mathrm{N}$ en su punto más meridional y $38^{\circ} 44^{\prime} \mathrm{N}$ en el más septentrional, lo que la localiza bajo el dominio de los climas subtropicales, esto es, es la franja de transición entre los climas de latitudes medias y los tropicales. A su vez ocupa la fachada suroccidental del continente europeo así como la fachada occidental de la cuenca mediterránea, lo que imprime un carácter mediterráneo a su clima. Gracias a esta posición, los vientos predominantes resultan del oeste, húmedos e inestables, proporcionando las precipitaciones invernales, que llegan más debilitadas a las zonas de interior y orientales.

El alto grado de fluctuación entre las precipitaciones registradas de unos años a otros, así como su elevado índice de irregularidad pluviométrica interanual determinan que las condiciones normales o habituales de aporte de agua se vean afectadas con frecuencia, convirtiendo a este territorio en uno de los de mayor riesgo potencial de sequía de toda España. Solo en la cuenca del río Guadalquivir, el diseño de los embalses se ha visto obligado a dimensionarse a fin de ajustarse a ciclos multianuales debido a la intensidad y duración de los fenómenos de sequía que pueden presentarse (Confederación Hidrográfica del Guadalquivir, 2007; Pita, 1998, 1989).

\subsection{Datos}

Para elaborar este estudio se ha empleado la base de datos de precipitación CRU TS v. 3.22 suministrada por la Climatic Research Unit (CRU) de la Universidad de East Anglia (New et al., 2002). Consiste en un grid de $0,5^{\circ}$ x $0,5^{\circ}$ de resolución obtenido a partir de datos de 1224 estaciones meteorológicas compiladas y actualizadas durante los últimos 30 años a nivel mundial. En este trabajo se han usado las medias mensuales de la variable precipitación para el periodo temporal 1901-2012.

La base de datos aúna tres tipos de variables: primarias, secundarias y derivadas. Las variables primarias contemplan la temperatura y precipitación media así como el rango de temperatura diurna. La calidad y validez de los datos primarios reside en que para ellos, a diferencia de los secundarios y derivados, se dispuso de suficiente información sobre las estaciones de base como para obtener los datos en rejilla directamente de los datos observacionales. De este modo, cada dato mensual del grid se basa en una interpolación de los datos de las estaciones disponibles. Apunta Goerlich Gisbert (2012) que a nivel regional los datos son más fiables cuanto mayor sea el nivel de agregación espacial considerado, pero aún así recomienda un examen directo de los mismos. La idoneidad de emplear esta base de datos para el análisis de series temporales de precipitación y aplicación de índices de sequía, respecto a otro tipo de fuente 
de información, como puede ser la recogida por estaciones, se debe a que la información que pudiera extraerse de estaciones proporcionaría una indicación del fenómeno objeto de estudio en un punto concreto del espacio, mientras que los datos de la CRU, al tratarse de datos en rejilla, relacionan lo observado en ese punto con un valor promedio para un área dada. De igual forma los datos de series temporales derivados de observaciones de estaciones no son necesariamente precisos ni homogéneos en el tiempo. En el estudio realizado por Goerlich Gisbert (2012) se llevó a cabo un examen de los 285 píxeles correspondientes al territorio que comprende la Península Ibérica, indicándose que la información de partida contenida en ellos es siempre suficiente para las variables precipitación y temperatura media.

Centrados en el área de estudio de este trabajo, la rejilla que corresponde a Andalucía consta de 38 celdas, de las cuales se ha tomado el punto central como punto representativo. Mediante la aplicación del software ArcGis, se han extraído las 38 series de precipitación mensual que abarcan de 1901 a 2012 y cubren la totalidad de Andalucía. En la Figura 1 se recogen los puntos de grid y el código asignado a cada uno de ellos. Además, estos puntos se corresponden con los usados por el Subsistema Clima y la Red de Información Ambiental (REDIAM) de la Junta de Andalucía.

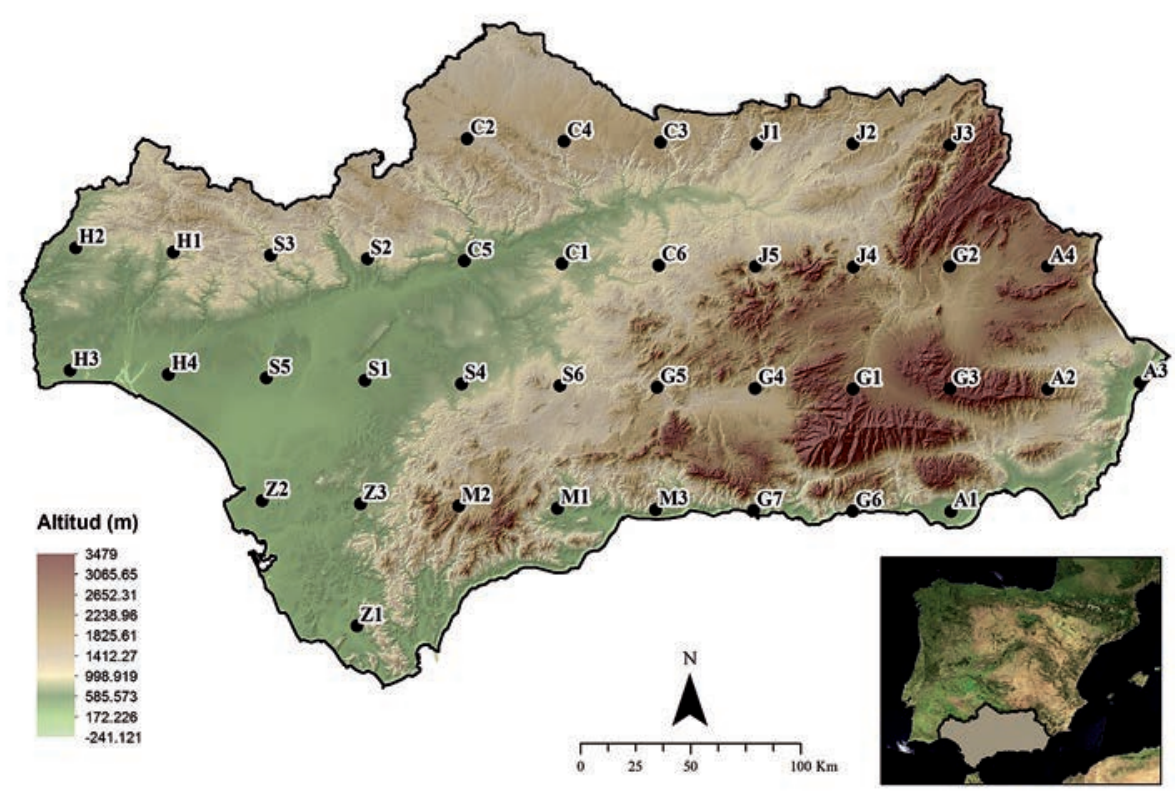

Figura 1. Localización e identificación de los puntos de grid. Las letras en la identificación de cada punto permiten conocer en qué ámbito provincial se inserta (Huelva (H), Sevilla (S), Cádiz (Z), Córdoba $(C)$, Málaga (M), Granada $(G)$, Jaén (J) y Almería (A)). 


\subsection{Análisis de variabilidad y tendencias aplicados al estudio de la precipitación}

Respondiendo al tercer objetivo específico planteado, se ha realizado un breve análisis sobre los principales estadísticos descriptivos de las series de precipitación, junto a pruebas de normalidad (Shapiro-Wilks) y cálculos del coeficiente de variación, definido como el cociente, en porcentaje, entre la desviación típica y el promedio de los totales anuales de precipitación para el periodo señalado, con el fin de analizar la variabilidad de las series de precipitación por subperiodos. Las tendencias de las precipitaciones han sido evaluadas mediante regresión lineal.

\subsection{Cálculo de índices de sequía}

Para caracterizar los eventos secos acontecidos históricamente se han utilizado tres índices diferentes de sequía, el Índice Estandarizado de Precipitación (SPI), el Índice Estandarizado de Precipitación y Evapotranspiración (SPEI), y el Índice Estandarizado de Sequía Pluviométrica (IESP).

\subsection{1. Índice Estandarizado de Precipitación (SPI)}

Este índice, formulado por McKee et al. (1993) y de aplicación extendida en todo el mundo, mide el déficit de precipitación para múltiples escalas temporales, de modo que se puedan tener en cuenta los impactos que un déficit pueda ocasionar sobre los recursos hídricos en función de la humedad del suelo, acumulación de nieve, escorrentía, aguas subterráneas y reservas de embalses.

El SPI se calcula a partir de la conversión de datos de precipitación de una serie lo suficientemente larga (mínimo 30 años) a probabilidades basadas en registros de precipitación acumuladas en 1, 2, 6, 12, 24 ó 48 meses, ajustando la función de distribución a una función adecuada. Dichas probabilidades componen series normalizadas con un promedio de 0 y una desviación estándar de 1 . A cada valor de la precipitación acumulada se le asigna un valor de la función, y se determina la probabilidad de ocurrencia de ésta, que coincide a su vez con la probabilidad de precipitación (SPI del dato de precipitación acumulada). El valor del SPI representa pues la probabilidad de ocurrencia de una determinada precipitación acumulada, siendo simplemente el número de veces que un valor concreto de la precipitación acumulada en un periodo temporal se separa de la media de la serie, medido en unidades de desviación típica.

Una sequía se produce siempre que el SPI sea negativo y alcance un valor menor que o igual a -0,99. El fenómeno finaliza cuando el SPI se hace positivo (Tabla 1). Cada fenómeno de sequía, por lo tanto, tiene una duración definida por su comienzo y su final, y una intensidad diferente para cada mes que dure el fenómeno (Garrido del Pozo et al., 2006).

La principal ventaja del SPI con relación a otros índices como puedan ser los desarrollados por Palmer en la década de los sesenta en Estados Unidos, es que permite el análisis de los impactos de las sequías en distintas escalas temporales. Además, puede identificar distintos tipos de sequías, ya que diferentes sistemas naturales y sectores económicos pueden responder a las condiciones de sequía en escalas de tiempo muy diferentes (Vicente-Serrano et al., 2012) 
Tabla 1. Clasificación de los valores del SPI. Fuente: National Drought Mitigation Center.

\begin{tabular}{|l|l|}
\hline \multicolumn{1}{|c|}{ Valores SPI } & \multicolumn{1}{c|}{ Clasificación } \\
\hline Mayor o igual a 2,00 & Extremadamente húmedo \\
\hline Entre 1,50 y 1,99 & Muy húmedo \\
\hline Entre 1,00 y 1,49 & Moderadamente húmedo \\
\hline Entre $-0,99$ y 0,99 & Aproximadamente normal \\
\hline Entre $-1,00$ y $-1,49$ & Moderadamente seco \\
\hline Entre $-1,50$ y $-1,99$ & Severamente seco \\
\hline Menor o igual a $-2,00$ & Extremadamente seco \\
\hline
\end{tabular}

En este trabajo se ha usado la distribución Gamma para transformar las series de precipitación a unidades estandarizadas, tal como se establece en la formulación original del índice. De igual forma, se ha usado la escala de 12 meses acumulados, más adecuada para una caracterización general de la sequía hidrológica, pues la acumulación en periodos más cortos derivaría en resultados indicativos de sequía meteorológica.

\subsection{2. Índice Estandarizado de Precipitación y Evapotranspiración (SPEI)}

El SPI, definido anteriormente, basa su cálculo únicamente en datos de precipitación, y no en otras variables que pueden influir en las condiciones de sequía. Otros índices de sequía suelen incluir datos de temperatura en su formulación (como el PDSI definido por Palmer), con la intención de identificar los impactos de este fenómeno en diferentes sistemas ecológicos, hidrológicos y agrícolas. Variables como la temperatura o la evapotranspiración potencial podrían proporcionan aportes adicionales al estudio de la sequía, por lo que podría resultar conveniente emplear índices que incorporan en su cálculo este tipo de variables. El SPEI, formulado por Vicente-Serrano et al. (2010) a partir del SPI, está basado tanto en registros de precipitación como en la evapotranspiración, y combina la sensibilidad a los cambios de demanda de evaporación con la sencillez de cálculo y la naturaleza multi-temporal del SPI. Los valores que toma el SPEI para la determinación de episodios secos son los mismos que el SPI (Tabla 1). Este índice ha sido ampliamente aceptado y utilizado en estudios de sequía a muy diversas escalas (Vicente-Serrano et al., 2014a; Manatsa et al., 2015; Naumann et al., 2015; Blauhut et al., 2015; Stagge et al., 2015a, 2015b; Das et al., 2016; Rhee and Cho, 2016; Yang et al., 2016).

Los valores del SPEI se han obtenido del Global SPEI Database, del Instituto Pirenaico de Ecología y de la Estación Experimental de Aula Dei del Consejo Superior de Investigaciones Científicas (CSIC), que a su vez se ha servido de la misma base de datos de la CRU empleada aquí, en el periodo temporal 1901-2012, para la estimación de la evapotranspiración potencial por el método de Penman-Monteith (Allen et al., 1998).

\subsection{3. Índice Estandarizado de Sequía Pluviométrica (IESP)}

Este es un índice mensual de sequía pluviométrica formulado por Pita (2001b) y basado en el cálculo de anomalías mensuales acumuladas. En este sentido es muy similar al SPI pues, al igual que en éste, las anomalías son estandarizadas, correspondiendo los valores negativos a meses secos y los valores positivos a meses húmedos. 
La esencia del IESP, así como su principal diferenciación respecto a otros índices similares, es que reinicia los cálculos de las anomalías acumuladas cada vez que se produce un nuevo mes seco en el marco de un periodo excedentario. Ello permite reflejar las secuencias secas de diferentes longitudes a partir de una única elaboración del índice, frente al SPI, que requiere una aplicación a múltiples escalas temporales para reflejar las diferentes duraciones de la sequía (Pita, 2001b). Los posibles valores del IESP quedan recogidos en la Tabla 2, donde se detallan los diferentes niveles o intensidades de sequía que pueden presentarse.

Tabla 2. Clasificación de los valores del IESP. Fuente: Pita (2001b).

\begin{tabular}{|l|l|}
\hline \multicolumn{1}{|c|}{ Valores IESP } & \multicolumn{1}{c|}{ Clasificación } \\
\hline IESP $\geq 0$ & Sin sequía \\
\hline$-1<$ IESP $\leq 0$ & Sequía moderada \\
\hline$-1,7<$ IESP $\leq-1$ & Sequía severa \\
\hline$-2,3<$ IESP $\leq-1,7$ & Sequía extraordinaria \\
\hline IESP $\leq-2,3$ & Sequía excepcional \\
\hline
\end{tabular}

El IESP es empleado desde la Consejería de Medio Ambiente y Ordenación del Territorio de la Junta de Andalucía para el seguimiento mensual de las sequías en el territorio andaluz. La utilización de este índice, en un área con elevada variabilidad interanual de las precipitaciones responde a la necesidad de identificar las secuencias secas registradas a partir de la sucesión de meses lluviosos y secos. Sánchez-Toribio et al. (2010) en un estudio sobre la demanda evaporativa y los requerimiento hídricos de los principales cultivos del valle del Guadalentín en periodos de sequía, emplean el IESP en la identificación de las secuencias secas por la precisión con la que refleja el comienzo y fin de cada secuencia, su duración e intensidad. Igualmente, hacen alusión a la efectividad de este índice en el análisis de las secuencias secas de larga duración, característica de los dominios mediterráneos, por la no acumulación de anomalías en su formulación.

En el análisis llevado a cabo se ha empleado únicamente la variable precipitación, pues en el medio mediterráneo, en el que se enmarca principalmente Andalucía, ésta constituye el elemento más variable, y en consecuencia es la responsable de la mayor parte de la variabilidad y génesis de las sequías (Capel-Molina, 1987). Los índices basados en datos de precipitación realizan un buen desempeño en comparación a índices más complejos (Pita, 2001a; Ayuso et al., 2015).

\subsection{Análisis de subperiodos temporales}

Una vez obtenidos los valores de los índices de sequía para las series consideradas en cada punto de rejilla, se ha realizado una división de las mismas en tres subperiodos temporales de 37 y 38 años, escogiendo las siguientes ventanas temporales: $w 1=1901$ 1937, w2 = 1938-1975 y w3 = 1976-2012.

Teniendo en cuenta que la variabilidad espacial del fenómeno de la sequía es previsiblemente inferior a la que caracteriza a la propia precipitación (Limones, 2013) se ha optado por aplicar diferentes test y análisis a los índices de sequía empleados. Además 
del ajuste de modelos lineales a las series temporales, se han usado diversos test y estadísticos no paramétricos, cuyos resultados son independientes de la distribución de probabilidad de los datos (González et al., 1999). También se ha aplicado el coeficiente de correlación de Pearson para comparar el comportamiento entre índices en cada uno de los distintos puntos de rejilla. De igual modo se ha aplicado entre los distintos subperiodos el test $t$ de diferencia de medias. Este test consiste en la comparación de la media como estadístico central entre dos muestras relacionadas para determinar si existe un cambio o diferencia entre ellas. La hipótesis nula $\left(\mathrm{H}_{0}\right)$ en este test consiste en la igualdad de las medias de dos subperiodos, lo que implicaría ausencia de cambio.

\section{Resultados y discusión}

\subsection{Precipitación. Tendencias y coeficientes de variabilidad}

La Figura 2 muestra el diagrama de cajas y bigotes de la precipitación anual en cada uno de los puntos de rejilla estudiados. En ella se pueden apreciar los valores medios de precipitación total anual, que se encuentran en torno a los 500-600 mm/año en la mayor parte de la geografía andaluza, a excepción de la región más oriental, donde los promedios rara vez alcanzan los $400 \mathrm{~mm} / \mathrm{año}$, junto a puntos en el interior del valle del Guadalquivir (puntos G1, G2, J1 y J2, en las provincias de Granada y Jaén) y regiones a sotavento de los regímenes de vientos del oeste (punto S2 en la Sierra Norte de Sevilla y G6 y G7, en la costa granadina) con cantidades anuales de precipitación inferiores debido a su localización geográfica. La región suroccidental andaluza se ve influenciada por los flujos de componente oeste, asociados a las masas de aire polar marítimo y tropical marítimo principalmente, así como por los vientos de componente oeste y noroeste que llegan al sur debilitados (Pita, 1998). En la Figura 2 puede también apreciarse la marcada variabilidad interanual del régimen de precipitación, la asimetría de la distribución de la precipitación anual, así como valores extremos por encima de $1200 \mathrm{~mm}$ /año correspondientes a tres zonas localizadas: por un lado el sur y suroeste de las provincias de Cádiz y Huelva respectivamente (Z1 y H3) que, por su disposición geográfica, reciben la entrada de las perturbaciones frontales a través del golfo de Cádiz; y por otra, el punto en el centro de la provincia de Málaga (M1).

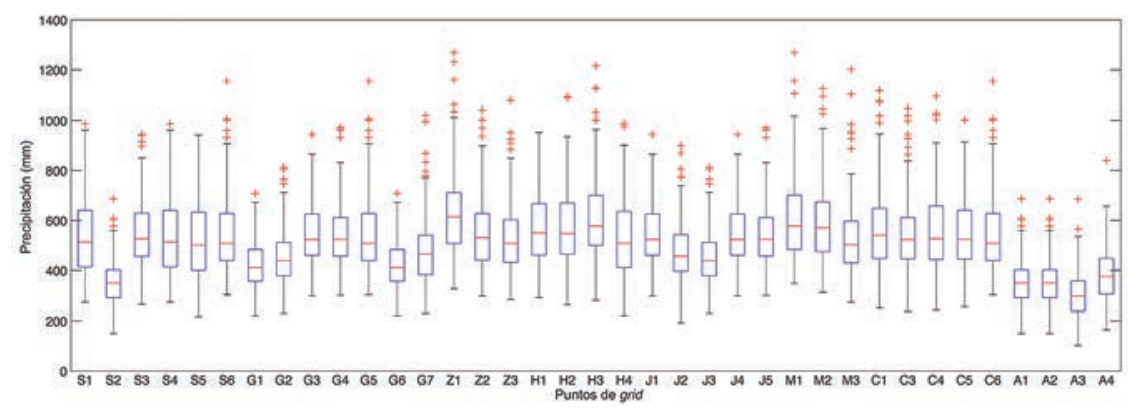

Figura 2. Diagrama de cajas y bigotes de la precipitación anual para las series de precipitación en el periodo 1901-2012. Se señalan los valores correspondientes a la mediana (rojo), percentiles 25 y 75 (extremos inferior y superior de las cajas azules) y los percentiles 2,5 y 97,5 (extremos inferior y superior de las líneas discontinuas negras). Las cruces en rojo indican valores extremos o anómalos. 
La pendiente obtenida en el análisis de regresión proporciona una estimación de las tendencias en las series de precipitación. La Figura 3 muestra el valor de dichas tendencias (en mm/año) para el periodo completo, pudiendo determinarse diferencias entre los distintos puntos o regiones. En general se observa una tendencia negativa para la mayor parte de Andalucía. Sectorialmente, el decrecimiento de la precipitación es poco marcado (entre 0 y - 0,5 mm/año) en el valle del Guadalquivir, el litoral malagueño y granadino así como en la comarca almeriense. Esta tendencia se hace positiva en prácticamente toda el área onubense (con valores por encima de $0,5 \mathrm{~mm} / \mathrm{año} \mathrm{y} \mathrm{solo} \mathrm{significativa} \mathrm{al} \mathrm{nivel}$ de confianza del $95 \%$ en el punto $\mathrm{H} 2$ ) y gaditana (con valores bajos). Los valores que indican un descenso más acusado $(<-0,5 \mathrm{~mm} / \mathrm{año})$ de las precipitaciones se han localizado en la totalidad de la provincia de Jaén, Sierra Nevada y Sierra de Baza, el valle medio del Guadalquivir, la Sierra del Estrecho y los Alcornocales, llegando a indicar tendencias negativas significativas al nivel de confianza del $95 \%$.

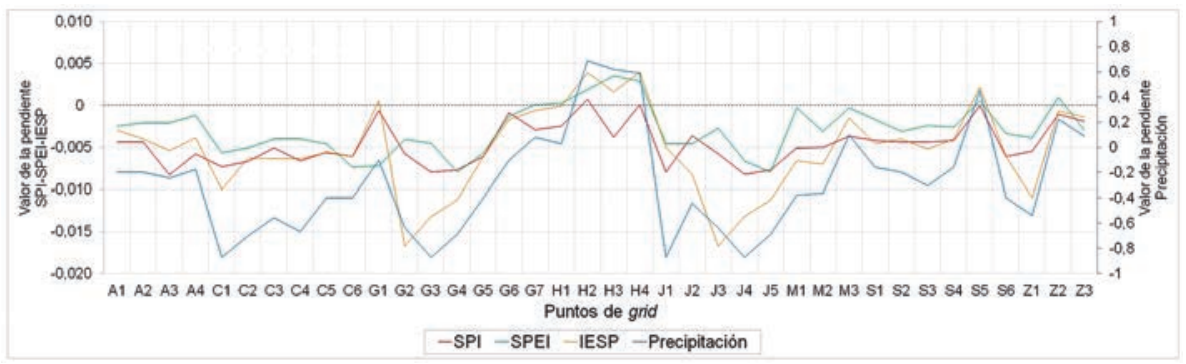

Figura 3. Tendencias de las series de precipitación anual (mm/año) y de los índices IESP, SPI y SPEI, en cada uno de los puntos analizados obtenidas a partir de la pendiente de la recta de regresión frente al tiempo en el periodo 1901-2012.

A pesar de la existencia de una tendencia negativa generalizada para la precipitación, la presencia de valores positivos denota un comportamiento no uniforme del conjunto regional, de acuerdo con lo señalado por Camarillo (1997) a partir de las comparativas realizadas entre distintas regiones peninsulares y mediterráneas. Estos resultados concuerdan con los obtenidos por Ruiz Sinoga et al. (2011) en su estudio de la evolución de las tendencias de la precipitación anual en el Mediterráneo andaluz, en el que señalan un descenso poco significativo en las estaciones localizadas más al sureste como Almería o Motril e incluso el litoral malagueño. Los cambios hacia una tendencia negativa resultan significativos, según se señala también en este estudio, en las estaciones de montaña como las de Sierra Nevada, Antequera y Gaucín.

En la Figura 4 se muestra el análisis del coeficiente de variación de los totales anuales de precipitación, representado cartográficamente para toda la región de estudio, interpolando los resultados a partir de los valores calculados para los 38 puntos de rejilla y para cada una de las tres ventanas temporales consideradas (w1 = 1901-1937, w2 = 1938-1975 y w3 = 1976-2012). En esta figura puede observarse cómo la variabilidad en las precipitaciones ha sido mayor en los dos últimos subperiodos, alcanzándose valores de precipitación máximos y/o mínimos superiores al subperíodo anterior. Así, en 
el primer subperíodo, la variación fue del $30 \%$ en casi la totalidad del territorio con la excepción de variaciones inferiores en el norte y noreste de las provincias de Córdoba y Jaén, respectivamente. En el segundo subperíodo, el coeficiente de variación muestra, en general, un aumento en toda la región, particularmente más elevado en el noreste de Córdoba y la Sierra de Cazorla, Segura y las Villas. En el último subperíodo, la variabilidad de la precipitación crece en los extremos occidental y oriental de la región así como en el valle medio del Guadalquivir, y en particular en la costa malagueña, mientras que desciende en la provincia de Córdoba y en el noreste de Andalucía.

Estos resultados basados en el análisis de datos de rejilla global pueden verse afectados por el número y localización de las estaciones de medida usadas, que para distintos subperiodos podría no ser constante, lo que podría inducir cambios espurios de la varianza (Beguería et al., 2015). Aunque el análisis detallado de la precipitación estacional queda fuera del alcance de los objetivos de este trabajo, los resultados obtenidos del análisis de las variaciones interanuales de la precipitación están de acuerdo con los encontrados en otros trabajos sobre el tema basados en registros instrumentales (García-Barrón, 2007; GarcíaBarrón et al., 2011). De acuerdo con García-Barrón (2007) es evidente y previsible el incremento de la variabilidad de la precipitación junto con la disparidad interanual, señalando que serán más probables años de precipitación muy escasa frente a otros muy lluviosos, y posiblemente, en años consecutivos. Además, al igual que en los resultados presentados, este autor encuentra que en el último subperíodo, desde la década de los 70, se desarrolla una fase marcadamente ascendente de variabilidad hasta la actualidad.

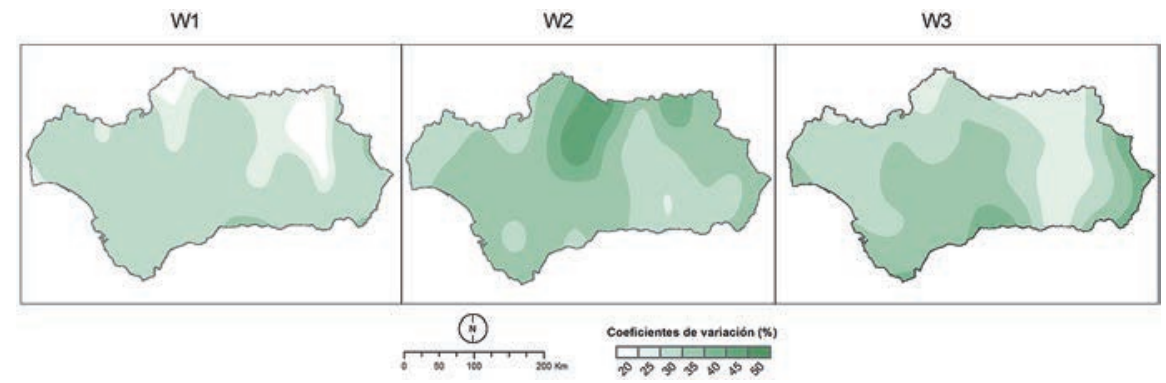

Figura 4. Coeficientes de variación de los totales anuales de precipitación (\%) interpolados espacialmente para los tres subperiodos considerados $(w 1, w 2$ y w3).

\section{2. Índices de sequía. Periodos secos y húmedos}

En este apartado se realiza el análisis de los índices de sequía considerados. En primer lugar, con el fin de analizar la similitud entre índices, se ha llevado a cabo un análisis de correlaciones entre los distintos pares de índices. A partir de la obtención del coeficiente de correlación de Pearson ( $r$ ), se ha comprobado la relación entre los diferentes índices. Para ello, en la Figura 5 se muestra dicho coeficiente calculado a partir de los valores de los distintos índices de sequía para los tres subperiodos considerados, en cada uno de los 38 puntos de rejilla.

Atendiendo al par de índices de sequía considerados y al subperíodo temporal analizado, la correlación entre los índices muestra importantes diferencias. Para el caso de 
los índices SPEI y SPI estos presentan correlaciones bastante similares para los tres subperiodos, alcanzando los valores máximos de correlación $\left(r_{\max }=0,87\right)$ en el segundo subperíodo para casi todos los puntos de rejilla. Cabe destacar el punto $\mathrm{S} 2$, correspondiente a una zona de transición entre la vega y la Sierra Norte de Sevilla, en el que la correlación entre ambos índices desciende considerablemente $(r=0,11)$ en el primer subperíodo, así como menores correlaciones en puntos del suroeste de la provincia de Sevilla durante el segundo subperíodo, (S5, $r=0,36)$ o el este de la provincia de Granada (G5, $r=0,38)$. La correlación media entre ambos es $r_{\text {mean }}=0,7$, lo que de forma aproximada indicaría la existencia de una fracción relevante de información contenida en un índice pero no en el otro.

Las correlaciones entre los índices SPI e IESP así como entre los índices SPEI e IESP son considerablemente más bajas que las obtenidas para el par de índices anteriores. La correlación media entre el SPI y el IESP es $r_{\text {mean }}=0,46$ mientras que entre el SPEI y el IESP es $r_{\text {mean }}=0,59$.

El IESP correlaciona peor con el SPI que con el SPEI, con correlaciones máximas mayores en el caso del SPEI $\left(r_{\max }=0,74\right)$ que en el SPI $\left(r_{\max }=0,67\right)$.

Si bien se ha observado una elevada correlación entre el SPI y el SPEI en la mayoría de los puntos de rejilla, diversos autores (Vicente-Serrano et al., 2012, 2014b) ponen de manifiesto la mayor idoneidad del SPEI para realizar monitorizaciones de las condiciones de sequía a escala global, dada la mejor capacidad (al incluir la variable evapotranspiración potencial, $\mathrm{ET}_{\mathrm{o}}$ ) para detectar los cambios de la demanda de evaporación por parte de la atmósfera, en particular, en áreas como la Península Ibérica.

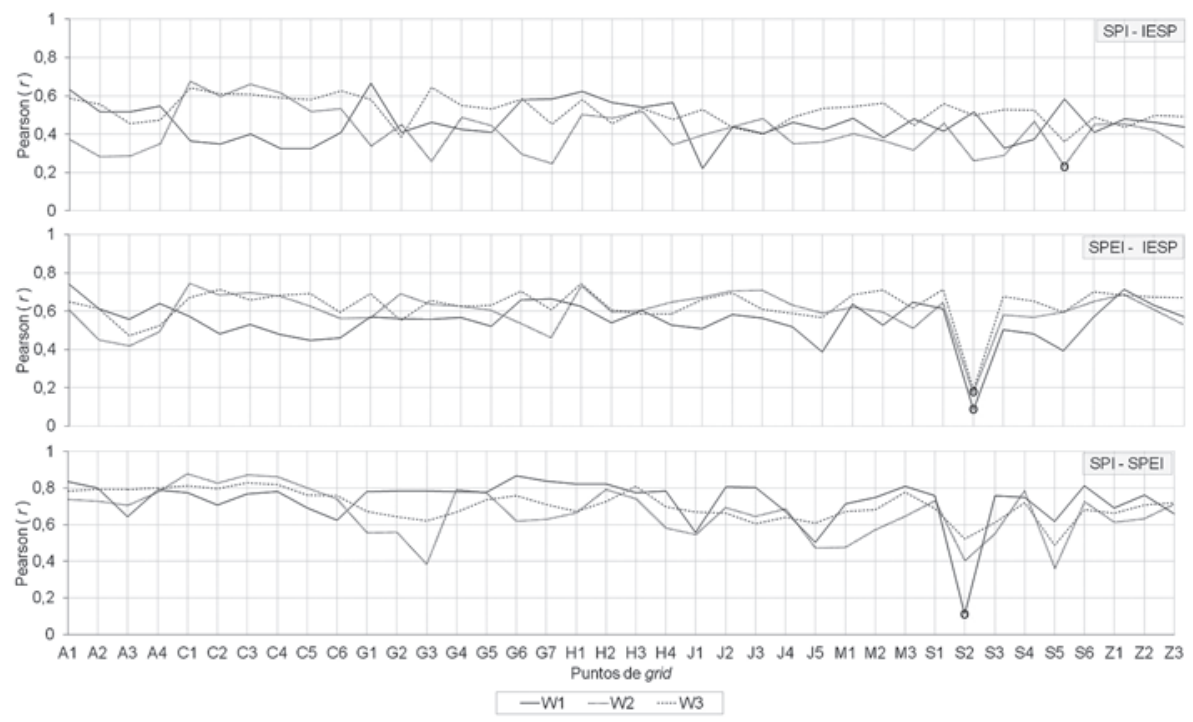

Figura 5. Valores del coeficiente de correlación de Pearson calculados a partir de los pares de valores de los índices SPI, SPEI e IESP en el periodo temporal total para cada uno de los tres subperiodos considerados $(w 1, w 2$ y w3). Los valores marcados con un círculo no son significativos al nivel de confianza del 95\% (teniendo en cuenta el número de grados de libertad efectivo en la correlación). 
En la Figura 6 se muestran los resultados obtenidos para los índices IESP, SPI y SPEI, en ocho puntos de rejilla (S3, G5, Z3, H1, J1, M2, C3, A1) representativos de la región. En general, los tres índices concuerdan en marcar los mismos periodos secos, con algunas diferencias en la intensidad y duración de los mismos. Así, por ejemplo, puede apreciarse en la mayoría de los puntos representados que el periodo seco a partir de 1981 es más largo y homogéneo en el IESP. En el primer subperíodo, atendiendo fundamentalmente a este índice, en Sevilla (S3), se detecta un largo periodo desde 1901 a 1937 con alternancia de constantes periodos cortos secos $(<-1,5)$ y húmedos de baja intensidad. Se ha registrado un periodo seco de 36 meses a final de este subperíodo que comienza alrededor de octubre de 1936 y finaliza en agosto de 1939. Granada (G5) y Málaga (M2) presentan un comportamiento relativamente similar con un leve periodo seco inicial que comienza en 1907, de intensidad inferior a -1,5 y una duración máxima de 20 meses. Huelva (H1), por su parte, no registra ningún periodo seco importante hasta finales de la década de los años 20, momento en el cual se produce una fuerte sequía que comienza en 1928 y finaliza una década más tarde con una intensidad máxima que alcanza el valor -4. En Cádiz (Z3) se produce un periodo seco destacable que comienza aproximadamente en 1903 y finaliza en 1910, con

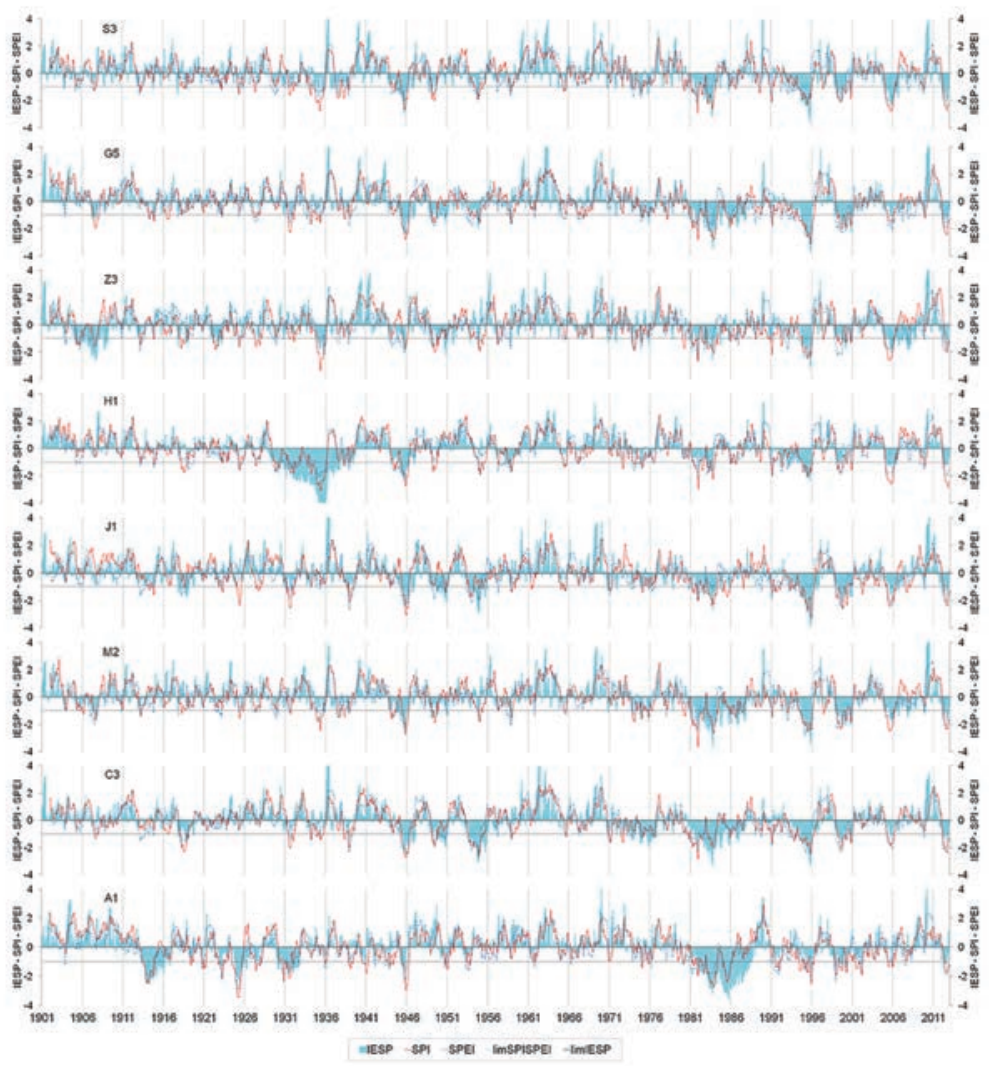

Figura 6. Valores de los índices IESP, SPI y SPEI calculados para distintos puntos de rejilla. Las líneas discontinuas y continuas indican el valor por debajo del cual se considera sequía para el IESP y el SPI-SPEI, respectivamente. 
intensidades máximas de -2. En Almería (A1) se producen tres periodos secos entre los años 1913-1916, 1924-1927 y 1929-1933, con intensidades que llegan a ser superiores a -2. En Córdoba y Jaén se produce un periodo seco que abarca desde 1917 a 1921.

Respecto al segundo subperíodo, w2, en todas las series, con mayor o menor intensidad, se detectan periodos secos en la primera mitad de la treintena, destacando un breve periodo seco en torno a 1946, presente en todos los puntos analizados. Todos los índices muestran que las décadas de los 60 y 70 se caracterizan por predominio de años húmedos y periodos secos cortos y moderados en todos los puntos analizados.

En el tercer subperíodo, w3, a partir de 1980, los índices muestran la aparición de importantes periodos secos en todos los puntos. Destaca la primera mitad de los 80, en la que se encuentra una prolongada sequía que perdura hasta 1988 en Granada, Málaga, Almería y en menor medida, en Córdoba. Los años 95 y 96 se caracterizan por ser muy secos, alcanzándose valores del IESP extremos (incluso mínimos) en Sevilla, Granada, Málaga, Cádiz y Jaén. El SPI y el SPEI detectan también estos periodos, pero en esta escala temporal tienden a mostrar duraciones de los mismos algo más cortas.

En cuanto a las tendencias referidas a los índices de sequía hay que señalar que los tres índices (Fig. 3) responden de forma similar, si bien es destacable notar ciertas singularidades. En general, el SPEI registra tendencias negativas (y no significativas al nivel de confianza del $95 \%$ en la mayoría de los puntos analizados) menos acusadas que el SPI o el IESP. El IESP, por su parte, muestra tendencias negativas significativas al nivel de confianza del $95 \%$ en un elevado número de puntos, más marcadas en los puntos G3, G4, G5, J4, J5 y Z2, con un comportamiento muy parecido al de la precipitación.

La aplicación del test $t$ de diferencia de medias permite evaluar cambios significativos en la media de los distintos índices de sequía entre los distintos periodos de tiempo considerados. La Tabla 3 muestra los resultados de este test en los ocho puntos de rejilla representativos de la región; en ella los cambios significativos al nivel de confianza del $95 \%$ se indican con un *. En general predomina una disminución de las medias a medida que se consideran periodos más cercanos al presente. Para el IESP, en la mayoría de los casos se tienen diferencias negativas significativas entre un periodo y el anterior, indicando una mayor presencia y/o intensidad de la sequía. Resultados análogos se obtienen para el SPI y el SPEI. Por otro lado, para el SPEI, no se detectan cambios significativos entre los periodos w1 y w2 en la mayoría de las puntos considerados, aunque si hay descensos significativos del índice cuando se considera el último periodo w3.

Por último, con el fin de aportar una caracterización general de la sequía en el área de estudio, la Figura 7 presenta los resultados del proceso de interpolación realizado para los tres índices considerados mediante el método Spline por subperiodos. Para los subperiodos w1 (1901-1937) y w2 (1938-1975), destaca el valor positivo de los tres índices para casi toda la región, indicando un predominio de las condiciones húmedas, excepto en el oeste y en el litoral mediterráneo; por el contrario, en el último subperíodo (1976-2012) 
Tabla 3. Resultados del test $t$ de diferencia de medias entre los subperiodos de tiempo considerados (w1, w2 y w3) del periodo temporal total 1901-2012, en los ocho puntos de rejilla seleccionados. Las diferencias significativas al nivel de confianza del 95\% se indican con un asterisco $(*)$.

\begin{tabular}{|c|c|c|c|c|}
\hline Punto de rejilla & Subperiodos & SPEI & SPI & IESP \\
\hline \multirow{3}{*}{ S3 } & w2-w1 & $0,248^{*}$ & $0,274 *$ & $-0,010$ \\
\hline & w3-w1 & $-0,229 *$ & $-0,238^{*}$ & $-0,395^{*}$ \\
\hline & w3-w2 & $-0,478 *$ & $-0,512 *$ & $-0,386^{*}$ \\
\hline \multirow{3}{*}{ G5 } & w2-w1 & 0,061 & 0,083 & $-0,061$ \\
\hline & w3-w1 & -0,409* & $-0,495^{*}$ & $-0,584 *$ \\
\hline & w3-w2 & $-0,470 *$ & $-0,578^{*}$ & $-0,523 *$ \\
\hline \multirow{3}{*}{$\mathrm{Z3}$} & w2-w1 & $-0,054$ & $0,367 *$ & $0,299 *$ \\
\hline & w3-w1 & -0,454* & $-0,123$ & $-0,143^{*}$ \\
\hline & w3-w2 & $-0,400 *$ & $-0,490^{*}$ & $-0,442 *$ \\
\hline \multirow{3}{*}{ H1 } & w2-w1 & $0,372 *$ & $0,297 *$ & $0,443^{*}$ \\
\hline & w3-w1 & $0,130^{*}$ & $-0,026$ & $0,202 *$ \\
\hline & w3-w2 & $-0,242 *$ & $-0,323^{*}$ & $-0,241^{*}$ \\
\hline \multirow{3}{*}{$\mathrm{J} 1$} & w2-w1 & 0,072 & $-0,098$ & $-0,211 *$ \\
\hline & w3-w1 & $-0,441^{*}$ & $-0,616^{*}$ & $-0,506^{*}$ \\
\hline & w3-w2 & $-0,514 *$ & $-0,518^{*}$ & $-0,295^{*}$ \\
\hline \multirow{3}{*}{ M2 } & w2-w1 & 0,066 & $0,121 *$ & $-0,084$ \\
\hline & w3-w1 & $-0,280^{*}$ & $-0,403^{*}$ & $-0,644 *$ \\
\hline & w3-w2 & $-0,346^{*}$ & $-0,524 *$ & $-0,563^{*}$ \\
\hline \multirow{3}{*}{$\mathrm{C} 3$} & w2-w1 & 0,044 & 0,004 & $-0,333 *$ \\
\hline & w3-w1 & $-0,301 *$ & $-0,420 *$ & $-0,635^{*}$ \\
\hline & w3-w2 & $-0,345^{*}$ & $-0,424 *$ & $-0,302 *$ \\
\hline \multirow{3}{*}{ A1 } & w2-w1 & $0,206^{*}$ & 0,033 & $0,364 *$ \\
\hline & w3-w1 & $-0,130 *$ & $-0,341^{*}$ & $-0,301 *$ \\
\hline & w3-w2 & $-0,336^{*}$ & $-0,375^{*}$ & $-0,670^{*}$ \\
\hline
\end{tabular}

los tres índices indican un predominio de las condiciones secas. En los tres subperiodos, el SPI es el índice que muestra un patrón espacial menos contrastado, mientras que el SPEI y el IESP presentan un gradiente zonal, que en el subperíodo w1, varía desde el extremo más occidental (condiciones más secas) hasta el centro-oeste de la región (condiciones más húmedas), se debilita en el subperíodo w2 y se invierte en el w3. 


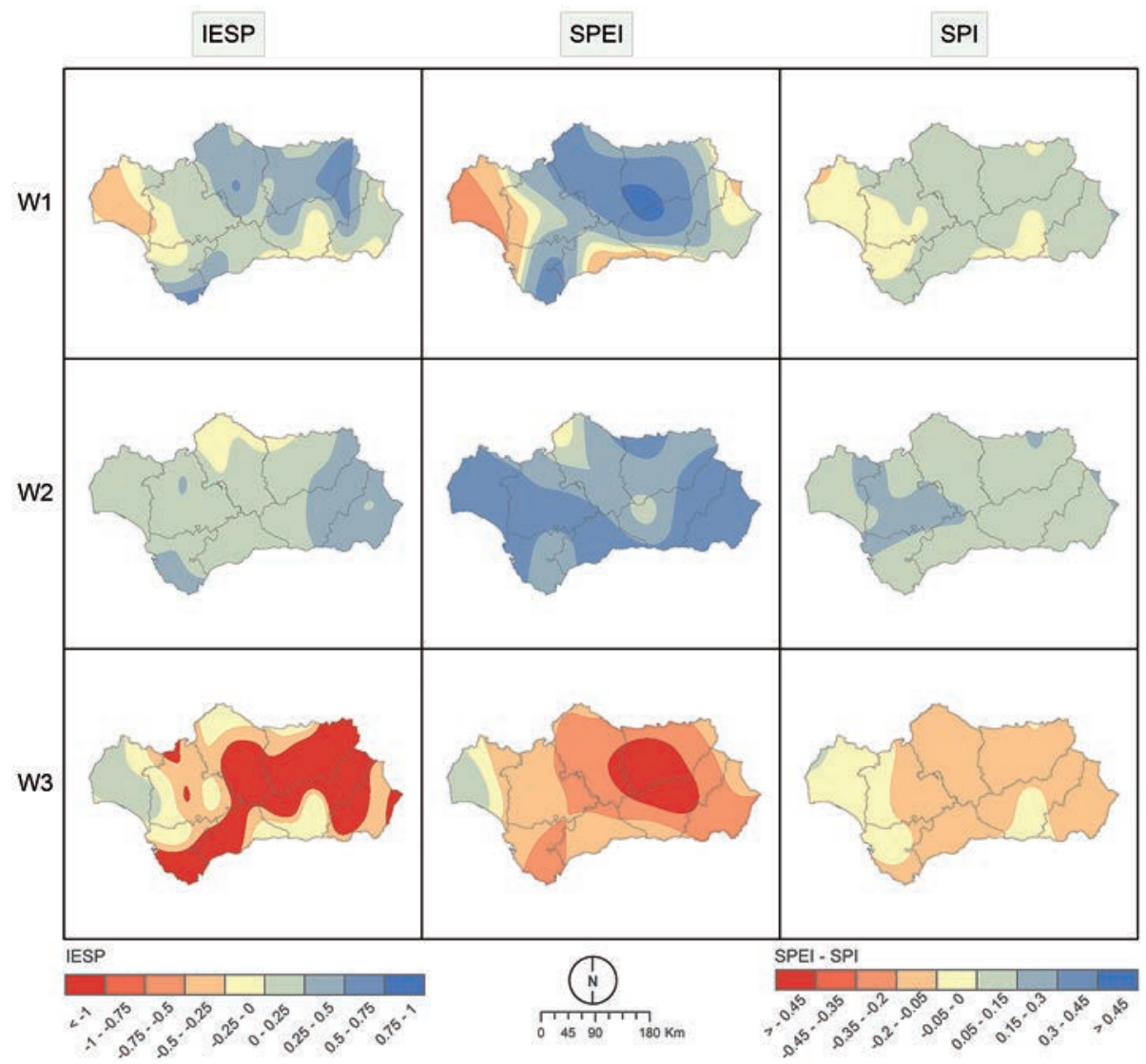

Figura 7. Mapas con los valores de los índices IESP, SPI y SPEI, promediados para cada subperíodo (w1, w2 y w3), resultantes del proceso de interpolación espacial mediante el método Spline.

\section{Conclusiones}

En el presente trabajo se ha realizado un análisis sobre el comportamiento y evolución de la precipitación y de diversos índices de sequía en Andalucía en el periodo 19011912, que ha permitido obtener resultados referidos al comportamiento del fenómeno de la sequía en dicha región. De este análisis se han extraído una serie de conclusiones que arrojan cierta perspectiva sobre el problema asociado a la escasez actual de recursos hídricos en la región sur de la Península Ibérica, en un contexto de elevada demanda hídrica por diversos sectores socioeconómicos como la agricultura o el turismo (Hidalgo-Muñoz et al., 2015).

Así, en cuanto a la variabilidad de las precipitaciones resalta el hecho de la baja variabilidad de la precipitación en el primer subperíodo analizado, 1901-1937, y el progresivo incremento de la misma en los sucesivos subperiodos, 1938-1975 y 1976-2012. 
Este incremento de la variabilidad se hace patente sobre todo en el último subperíodo en los extremos occidental y oriental de la región, así como en la costa malagueña. Los cambios encontrados en la variabilidad de la precipitación en Andalucía, podrían estar asociados con cambios en la variabilidad de la Oscilación del Atlántico Norte, principal responsable de la precipitación en la región durante el periodo más lluvioso del año (Esteban-Parra et al., 1998).

La precipitación muestra además tendencias negativas en gran parte de Andalucía. El decrecimiento es menos señalado (entre 0 y - 0,5 mm/año) en el valle del Guadalquivir, el litoral malagueño y granadino así como en la región almeriense, mientras que los descensos más acusados de las precipitaciones ( $<-0,5 \mathrm{~mm} / \mathrm{año})$ se han localizado en la totalidad de la provincia de Jaén, Sierra Nevada y Sierra de Baza, el valle medio del Guadalquivir, la Sierra del Estrecho y los Alcornocales. Nuevamente se hace constar el comportamiento espacialmente no uniforme del conjunto regional de Andalucía lo que concuerda con la regionalización de la precipitación en la Península Ibérica, en la que el sudeste peninsular conforma un modo de variabilidad característico de la precipitación (Esteban-Parra et al., 1998).

Los índices de sequía por su parte muestran diferencias en la identificación el de los periodos secos. En general, el SPI y el SPEI presentan evoluciones temporales similares, de tal forma que para la mayoría de las series analizadas, la correlación temporal entre estos índices es elevada y significativa, salvo en el norte de Sevilla. El IESP presenta valores más bajos de correlación con estos índices, destacando las bajas correlaciones en el último periodo de tiempo. Debe tenerse en cuenta que tanto el SPI como el IESP no consideran los cambios en la demanda atmosférica de humedad (evapotranspiración potencial, ET ), a pesar de que estudios recientes han encontrando tendencias significativas en la ET que afectan a las condiciones de sequía en la Península Ibérica (Vicente-Serrano et al., 2014b).

Los periodos secos históricos más importantes corresponden al inicio de la década de los ochenta con valores del índice IESP de -4,3 en Almería; a comienzos de la década de los noventa con valores de -4,63 en Granada, y por último, la sequía del periodo 2008-2012, que alcanzó valores de -2,84 en puntos de Jaén.

Así pues, aunque los descensos en precipitación encontrados son moderados, parece constatarse un aumento significativo de la incidencia de la sequía en Andalucía, de acuerdo con el descenso en los caudales encontrados en Lorenzo-Lacruz et al. (2012) e Hidalgo-Muñoz et al. (2015) en las cuencas del Guadalquivir y Mediterránea sur.

Dadas las importantes repercusiones de la sequía, más aun en una zona de alta sobrexplotación hídrica, el análisis de índices como los aquí considerados, que permiten una identificación de los eventos de sequía, puede ayudar a la monitorización y predicción de la misma.

\section{Agradecimientos}

El Ministerio de Economía y Competitividad, con financiación adicional de la Comunidad Europea (Fondos FEDER), proyecto CGL2013-48539-R y el Gobierno 
Regional Andaluz, proyecto P11-RNM-7941, han financiado este estudio. Agradecemos a los revisores sus útiles y apropiados comentarios, que han ayudado a mejorar el artículo.

\section{Referencias}

Allen, R.G., Pereira, L.S., Raes, D., Smith, M. 1998. Crop evapotranspiration. Guidelines for computing crop water requirements. FAO Irrigation and drainage paper 56. FAO, Rome, 300 (9), D05109.

American Meteorological Society 2004. Statement on meteorological drought. Bulletin of American Meteorological Society 85, 771-773.

Ayuso, J.L., Ayuso-Ruiz, P., García-Marín, A.P., Estévez, J., Taguas, E.V. 2015. Local Analysis of the Characteristics and Frequency of Extreme Droughts in Málaga Using the SPI (Standardized Precipitation Index). En J.L.A. Muñoz, J.L.Y. Blanco, S.F. Capuz-Rizo (eds.), Project Management and Engineering, Lecture Notes in Management and Industrial Engineering. Springer International Publishing, pp. 167-179.

Beguería, S., Vicente-Serrano, S.M., Reig, F., Latorre, B. 2014. Standardized precipitation evapotranspiration index (SPEI) revisited: parameter fitting, evapotranspiration models, tools, datasets and drought monitoring. International Journal of Climatology 34, 3001-3023.

Beguería, S., Vicente-Serrano, S.M., Tomás-Burguera, M., Maneta, M. 2015. Bias in the variance of gridded data sets leads to misleading conclusions about changes in climate variability. International Journal of Climatology. Doi: 10.1002/joc.4561.

Blauhut, V., Stahl, K., Stagge, J.H., Tallaksen, L.M., De Stefano, L., Vogt, J. 2015. Estimating drought risk across Europe from reported drought impacts, hazard indicators and vulnerability factors. Hydrology and Earth System Sciences Discussion 12, 12515-12566.

Burton, I., White, G.F., Kates, R.W. 1979. The environment as hazard. Oxford University Press, New York.

Camarillo, J.M. 1997. Evolución de la precipitación en Andalucía Oriental a partir de series instrumentales: los cambios en la variabilidad pluviométrica. Dpto. de Geografía Física y A.G.R., Universidad de Sevilla.

Ceballos, A. 2013. Análisis de la variabilidad espacio-temporal de las precipitaciones en el sector español de la cuenca del Duero (1961-2005). Boletín de la Asociación de Geógrafos Españoles 61, 235-259.

Confederación Hidrográfica del Guadalquivir 2007. Plan especial de actuación en situaciones de alerta y eventual sequía de la cuenca hidrográfica del Guadalquivir. Sevilla.

Cubillo, F., De Castro, J. 2007. Guía para la elaboración de planes de emergencia por sequía en sistemas de abastecimiento urbano. Ministerio de Medio Ambiente y Asociación Española de Abastecimientos de Agua y Saneamiento (Aeas).

Consejería de Medio Ambiente y Ordenación del Territorio. Disponible en: http: //www. juntadeandalucia.es /medioambiente/site/portalweb/

Das, P.K., Dutta, D., Sharma, J.R., Dadhwal, V.K. 2016. Trends and behaviour of meteorological drought (1901-2008) over Indian region using standardized precipitation-evapotranspiration index. International Journal of Climatology, 36, 909-916.

Del Río, S., Herrero, L., Fraile, R., Penas, A. 2011. Spatial distribution of recent rainfall trends in Spain (1961-2006). International Journal of Climatology 31, 656-667.

Edwards, D.C., McKee, T.B. 1997. Characteristics of 20th century drought in the United States at multiple time scales. Climatology Report N ${ }^{\circ}$ 97-2, Colorado State Univ., Fort Collins, CO. 
Esteban-Parra, M.J., Rodrigo, F.S., Castro-Díez, Y. 1998. Spatial and temporal patterns of precipitation in Spain for the period 1880-1992. International Journal of climatology 18, 15571574 .

Esteban-Parra, M.J., Pozo-Vázquez, D., Rodrigo, F.S., Castro-Díez, Y. 2003. Temperature and Precipitation Variability and Trends in Northern Spain in the Context of the Iberian Peninsula Climate. En H.J. Bolle (ed.), Mediterranean Climate, Regional Climate Studies. Springer, Berlin, pp. 259-276.

García-Barrón, L. 2007. La evolución climática del Suroeste de la Península Ibérica basada en registros instrumentales. En El Cambio climático en Andalucía: Evolución y consecuencias medioambientales, Consejería de Medio Ambiente (Junta de Andalucía), Sevilla, pp. 81-95.

García-Barrón, L., Aguilar, M., Sousa, A. 2011. Evolution of annual rainfall irregularity in the southwest of the Iberian Peninsula. Theoretical and Applied Climatology 103 (1), 13-26.

Garrido del Pozo, N., Mora García, M., Ortíz de Galisteo, J.P., Álvarez Alonso, J.P., Buendía Moya, G. 2006. Las sequías climáticas en la cuenca del Duero. En Congreso homenaje al Douro/Duero y sus ríos: Memoria, cultura y porvenir, Centro Meteorológico de Castilla y León MMAM, Zamora.

Gil, S., López, F. 2011. Tendencia de las precipitaciones y temperaturas en una pequeña cuenca fluvial del sureste peninsular semiárido. Boletín de la Asociación de Geógrafos Españoles 56, 349-371.

Goerlich Gisbert, F.J. 2012. Datos climáticos históricos para las regiones españolas. CRU TS 2.1. Investigaciones de Historia Económica 8, 29-40.

González, J.C., de Luis, M., Raventós, J., Sánchez, J.R., Cortina, J. 1999. Aproximación metodológica al análisis de la estructura de las tendencias de lluvia. Geographicalia 37, 53-72.

Hidalgo-Muñoz, J.M., Gámiz-Fortis, S.R., Castro-Díez, Y., Argüeso, D, Esteban-Parra, M.J. 2015. Long-range seasonal streamflow forecasting over the Iberian Peninsula using largescale atmospheric and oceanic information. Water Resources Research 51. Doi: 10.1002/ wrcr.v51.5, 3543-3567.

Jarraud, M. 2013. Hacia sociedades más resilientes frente a la sequía. En United Nations Convention to Combat Desertification. High-Level meeting on national drought policy. Génova.

Kangas, R.S., Brown, T.J. 2007. Characteristics of US drought and pluvial from a high-resolution spatial dataset. International Journal of Climatology 27, 1303-1325.

Limones, N. 2013. El estudio de la sequía hidrológica en el mediterráneo español. Propuesta de aplicación del índice estandarizado de sequía pluviométrica a las aportaciones hídricas. Tesis doctoral, Universidad de Sevilla, Sevilla.

Lorenzo-Lacruz, J., Vicente-Serrano, S.M., González-Hidalgo, J.C., López-Moreno, J.I., Cortesi, N. 2012. Evaluación regional de la respuesta hidrológica a las condiciones de sequía climática a distintas escalas temporales en la Península Ibérica. En Cambio climático. Extremos e impactos. $8^{\circ}$ Congreso Internacional de la Asociación Española de Climatología (AEC), Salamanca, pp. 819-829.

Manatsa, D., Mushore, T., Lenouo, A. 2015. Improved predictability of droughts over southern Africa using the standardized precipitation evapotranspiration index and ENSO. Theoretical and Applied Climatology. Doi: 10.1007/s00704-015-1632-6.

Marín, R.G., Schnabel, S., Parra, F.J.L., Fernández, M.P. 2012. Evolución de las precipitaciones en el suroeste de la Península Ibérica (Extremadura). Nimbus Revista de Climatología, Meteorología y Paisaje 29-30, 277-288.

Marzol, M.V. 2001. Las sequías meteorológicas: Frecuencia e intensidad de la sequedad en las Islas Canarias (España). En N. Grande, P. Arrojo , J. Martínez (coords.), Una cita europea con la nueva cultura del agua. La Directiva Marco. Perspectivas en Portugal y España, II Congreso Ibérico sobre Planificación y gestión de aguas, Zaragoza, pp. 1055-1065. 
McKee, T.B., Doesken, N.J., Kleist, J. 1995. Drought Monitoring with multiple time scales. En Ninth Conference on Applied Climatology, American Meteorological Society, Dallas, Texas, pp. 233-236.

McKee, T.B., Doesken, N.J., Kleist, J. 1993. The relationship of drought frequency and duration to time scales. En Eighth Conference on Applied Climatology, American Meteorological Society, Anaheim, California, pp. 233-236.

National Drought Mitigation Center. Disponible en: http://drought.unl.edu/MonitoringTools/ Climate DivisionSPI.aspx.

Naumann, G., Spinoni, J., Vogt, J., Barbosa, P. 2015. Assessment of drought damages and their uncertainties in Europe. Environmental Research Letters 10, 124013.

New, M., Lister, D., Hulme, M., Makin, I. 2002. A high-resolution data set of surface climate over global land areas. Climate Research 21, 1-25.

Pita, M.F. 1989. La sequía como desastre natural: Su incidencia en el ámbito español. Norba 6-7, 31-62.

Pita, M.F. 1998. El clima de Andalucía. En Geografía de Andalucía, Ariel, Barcelona, pp. 137174.

Pita, M.F., Camarillo, J.M., Aguilar, M. 1999. La evolución de la variabilidad pluviométrica en Andalucía y sus relaciones con el índice de la NAO. En I Congreso de la Asociación Española de Climatología (AEC), Oikos-Tau, Barcelona, pp. 399-408.

Pita, M.F. 2001a. Sequías en la cuenca del Guadalquivir. En Causas y consecuencias de las sequías en España, Instituto Universitario de Geografía de la Universidad de Alicante y Caja de Ahorros del Mediterráneo, Alicante, pp. 303-343.

Pita, M.F. 2001b. Un nouvel indice de sécheresse pour les domaines méditerranéens. Application au bassin du Guadalquivir (sudouest de l'Espagne). Publications de l'Association Internationale de Climatologie 13, 225-234.

Pozo-Vázquez, D. 2000. Causas físicas de la variabilidad espacio-temporal del clima de la Península Ibérica en escalas interanual o decadal: NAO y ENSO. Tesis doctoral, Universidad de Granada, Granada.

Rhee, J., Cho, J. 2016. Future Changes in Drought Characteristics: Regional Analysis for South Korea under CMIP5 Projections. Journal of Hydrometeorology 17, 437-451.

Rico y Sinobas, M. 1851. Memoria sobre las causas meteorológico-físicas que producen las constantes sequías de Murcia y Almería, señalando los medios de atenuar sus efectos. Imp. a cargo de D. S. Compagni, Madrid. Disponible en: http://bibliotecadigital.jcyl.es/es/consulta/registro. cmd?id=1404

Rodrigo, F.S., Esteban-Parra, M.J., Pozo-Vázquez, D., Castro-Díez, Y. 2000. Rainfall variability in southern Spain on decadal to centennial time scales. International Journal of Climatology 20, 721-732.

Rodrigo, F.S., Esteban-Parra, M.J., Pozo-Vázquez, D., Castro-Díez, Y. 1999. A 500-year precipitation record in Southern Spain. International Journal of Climatology 19, 1233-1253.

Ruiz-Sinoga, J.D., García-Marín, R., Gabarrón-Galeote, M.A., Martínez-Murillo, J.F. 2012. Analysis of dry periods along a pluviometric gradient in Mediterranean southern Spain. International Journal of Climatology 32, 1558-1571.

Ruiz Sinoga, J.D., García Marín, R., Martínez Murillo, J.F., Gabarrón Galeote, M.A. 2011. Precipitation dynamics in southern Spain: trends and cycles. International Journal of Climatology 31, 2281-2289.

Sánchez-Toribio, M.I., García-Marín, R., Conesa-García, C., López-Bermúdez, F. 2010. Evaporative demand and water requirements of the principal crops of the Guadalentín valley (SE Spain) in drought periods. Spanish Journal of Agricultural Research 8, 66-75. 
Stagge, J.H., Kohn, I., Tallaksen, L.M., Stahl, K. 2015a. Modeling drought impact occurrence based on climatological drought indices for Europe. Journal of Hydrology. Doi: 10.1016/j. jhydrol.2015.09.039.

Stagge, J.H., Tallaksen, L.M., Gudmundsson, L., Van Loon, A.F., Stahl, K. 2015b. Candidate Distributions for Climatological Drought Indices (SPI and SPEI). International Journal of Climatology 35, 4027-4040.

Vicente-Serrano, S.M., Beguería, S., López-Moreno, J.I. 2010. A Multi-scalar drought index sensitive to global warming: The Standardized Precipitation Evapotranspiration Index - SPEI. Journal of Climate 23, 1696-1718.

Vicente-Serrano, S.M., Camarero, J.J., Azorin-Molina, C. 2014a. Diverse responses of forest growth to drought time-scales in the Northern Hemisphere. Global Ecology and Biogeography 23, 1019-1030.

Vicente-Serrano, S.M., López-Moreno, J.I., Beguería, S., Lorenzo-Lacruz, J., Sánchez-Lorenzo, A., García-Ruiz, J.M., Azorín-Molina, C., Morán-Tejeda, E., Revuelto, J., Trigo, R., Coelho, F., Espejo, F. 2014b. Evidence of increasing drought severity caused by temperature rise in southern Europe. Environmental Research Letters 9, 044001.

Vicente-Serrano, S.M., Beguería, S., Lorenzo-Lacruz, J., Camarero, J.J., López-Moreno, J.I., Azorín-Molina, C., Revuelto, J., Morán-Tejeda, E., Sánchez-Lorenzo, A. 2012. Análisis comparativo de diferentes índices de sequía para aplicaciones ecológicas, agrícolas e hidrológicas. En $8^{\circ}$ Congreso Internacional de la Asociación Española de Climatología, Salamanca, pp. 679-689.

Wilhite, D.A., Glantz, M.H. 1985. Understanding the Drought Phenomenon: The Role of Definitions. Water International 10, 111-120.

Yang, M., Yan, D., Yu, Y., Yang, Z. 2016. SPEI-based spatio-temporal analysis of drought in Haihe River Basin from 1961 to 2010. Advances in Meteorology. http://dx.doi. org/10.1155/2016/7658015. 